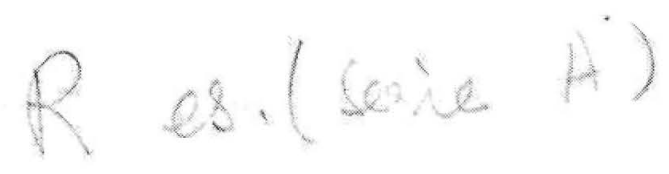

$616 \cdot 110$

\title{
sobre la influencia del tamaño de grano de la caliza en la formación de los mineralas del elínker
}

\author{
Hans Lehmann y Peter Thormann
}

\section{planteamiento del problema y realización del ensayo}

En el Congreso del otoño de 1961 de la Vereins Deutscher Zementwerke (Asociación de Fabricantes Alemanes de Cemento), se informó sobre las investigaciones que se ocuparon del comportamiento a la calcinación de las materias primas para la fabricación de cemento (1). Los resultados de las investigaciones condujeron, entre otras cuestiones, a aceptar que la finura de molienda del crudo -especialmente el tamaño del grano de la caliza-, así como la aptitud a la calcinación, el final de la formación del clínker y la iniciación en la clinkerización ejercen una influencia decisiva.

Por ello pareció indicado investigar la influencia de la finura teniendo en cuenta la composición química, la composición mineralógica y la temperatura de calcinación.

Para juzgar el comportamiento, las técnicas de investigación se refirieron a:

1. Determinación del contenido en $\mathrm{CaO}$ libre según Franke (2) y cálculo de la cantidad de cal combinada.

2. Determinación cuantitativa de los minerales del clínker mediante un recuento microscópico según secciones del clínker.

3. Determinación semicuantitativa de los minerales por medio del análisis estructural en rayos $\mathbf{X}$.

4. Investigaciones de la resistencia mecánica en pequeños prismas (3).

Para la preparación de las mezclas se emplearon las materias primas y las fracciones de la caliza, resumidas en la tabla 1 .

\section{Materias primas:}

Piedra caliza de Winterberg

Caolin Osmose de Zettlitz.

Illita de Illinois.

Polvo de cuarzo número 12, de Dörentrup.

$\mathrm{Fe}_{2} \mathrm{O}_{3}$ p. a.

\section{T A B L A 1 \\ Mezclas:}

Finura

1

2

3

4

5

6

Constitución caracteristica:

\begin{tabular}{|c|c}
$\begin{array}{c}\text { Fracción } \\
(\mu \mathrm{m})\end{array}$ & $\begin{array}{c}\text { Tamaño medio del grano } \\
(\mu \mathrm{m})\end{array}$ \\
$90-150$ & 100 \\
$60-90$ & 75 \\
$25-60$ & 40 \\
$5-20$ & 15 \\
$5-10$ & 7,5 \\
$0-5$ & 2
\end{tabular}

Tipo de mineral arcilloso / MF / finura

K

$2,2 \quad 1$

K $2,2 / 1$ 
La modificación de finura se limitó a la piedra caliza, por las siguientes razones:

1. El contenido en cal es esencialmente mayor que el de minerales de arcilla y cuarzo.

2. En la práctica habrá que limitarse a la molienda fina de la caliza, ya que los minerales de la arcilla, por lo general, son finos ya, y el componente cuarzo únicamente puede pulverizarse con un gasto extraordinario.

3. La mayor influencia en el final de la reacción, es de esperar que se deba al tamaño de grano de la caliza, ya que el $\mathrm{CaO}$ capaz de reaccionar se difunde entre los otros componentes de la reacción (4).

También es de esperar que la influencia del tamaño de grano de la caliza sobre los resultados de Ia investigación quede determinada decisivamente por la mutua influencia que ejercen otros factores. Por eso se modificó la composición químıca únicamente con respecto al módulo đe alúmina (MF). Además, se determinó la influencia del tipo de mineral arcilloso en ensayos paralelos. Las composiciones de las mezclas están indicadas en la tabla 2.

T A B L A 2

ANALISIS Y CONDICIONES DE ENSAYO

\begin{tabular}{|c|c|c|c|c|c|c|}
\hline \multirow{3}{*}{ Contenido $\{\%\}$} & \multicolumn{6}{|c|}{$\begin{array}{l}\text { Antilisis dol clinker } \\
\text { Serle do mezelos }\end{array}$} \\
\hline & & & & & & \\
\hline & K 2,2 & K 1,3 & K 0,5 & I 2,2 & 1,3 & 10,5 \\
\hline $\begin{array}{lllllllll}\mathrm{SiO}_{2} & \ldots & \ldots & \ldots & \ldots & \ldots & \ldots & \ldots\end{array}$ & 22,5 & 22,7 & 23,0 & 22,3 & 22,4 & 22,7 \\
\hline $\mathrm{Al}_{2} \mathrm{O}_{\mathrm{n}}+\mathrm{TiO}_{2} \ldots \ldots \ldots \ldots$ & 5,2 & 4,3 & 2,5 & 5,1 & 4,2 & 2,5 \\
\hline $\mathrm{Fe}_{2} \mathrm{O}_{2} \ldots \ldots \ldots \ldots$ & 2,3 & 3,3 & 5,1 & 2,3 & 3,3 & 5,1 \\
\hline $\begin{array}{cccccccc}\mathrm{CaO} & \ldots & \ldots & \ldots & \ldots & \ldots & \ldots & \ldots\end{array}$ & 67,5 & 67,7 & 68,0 & 66,8 & 66,8 & 67,5 \\
\hline $\begin{array}{lllll}\text { No dosificado } & \ldots & \ldots & \ldots & \ldots\end{array}$ & 2,5 & 2,2 & 1,4 & 3,5 & $\mathbf{3 , 3}$ & 2,1 \\
\hline Standard de cal $\ldots \ldots \ldots \ldots$ & 96 & 96 & 96 & 96 & 96 & 96 \\
\hline Módulo de silicatos $\ldots . .$. & 3,0 & 3,0 & 3,0 & 3,0 & 3,0 & 3,0 \\
\hline Módulo de fundentes ... & 2,2 & 1,3 & 0,5 & 2,2 & 1,3 & 0,5 \\
\hline
\end{tabular}

\begin{tabular}{|c|c|c|c|c|c|c|}
\hline \multirow[b]{2}{*}{ Composición minoralogica ( $\%$ ) } & \multicolumn{6}{|c|}{ Composteión potencial, según Bogü } \\
\hline & K 2,2 & K 1,3 & & & I 1,3 & 10,5 \\
\hline 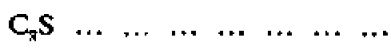 & 66,15 & 70,15 & 76,49 & 65,09 & 68,82 & 76,36 \\
\hline $\begin{array}{cccccccc}\mathrm{C}_{2} \mathrm{~s} & \ldots & \ldots & \ldots & \ldots & \ldots & \ldots & \ldots\end{array}$ & 14,50 & 11,98 & 8,14 & 14,80 & 12,34 & 7,54 \\
\hline $\begin{array}{cccccccc}C_{\mathrm{g}} \mathbf{A} & \ldots & \ldots & \ldots & \ldots & \ldots & \ldots & \ldots\end{array}$ & 9,72 & 5,75 & 0,00 & 9,60 & 5,67 & 0,00 \\
\hline $\begin{array}{lllllll}\mathbf{C}_{4} \mathbf{A F} & \ldots & \ldots & \ldots & \ldots & \ldots & \ldots\end{array}$ & 7,11 & 9,96 & 12,09 & 7,05 & 9,88 & 12,09 \\
\hline $\begin{array}{llllllll}\mathbf{C}_{8} \mathbf{F} & \ldots & \ldots & \ldots & \ldots & \ldots & \ldots & \ldots\end{array}$ & 0,00 & 0,00 & 1,93 & 0,00 & 0,00 & 1,87 \\
\hline
\end{tabular}

Condiciones constantes para todks las series:

Mezclas: Mezcladora por gravedad y tamizado.

Granulación: A mano.

Calcinación: En atmósfera oxidante en horno de Super-Kanthal.

Enfriamiento: Gránulos vertidos y enfriados con aire comprimido.

Trituración: Machacadora de mandibulas de laboratorio.

Velocidad de calentamiento: $5-8^{\circ} \mathrm{C} /$ minuto,

Tiempo de calentamiento: Treinta minutos. 


\section{resultados de la investigación}

\section{1. determinación del contenido en CaO libre}

Las determinaciones se realizaron según el método de Franke, y los resultados se representaron gráficamente en las figuras 1 y 2.

De ellas se deduce:

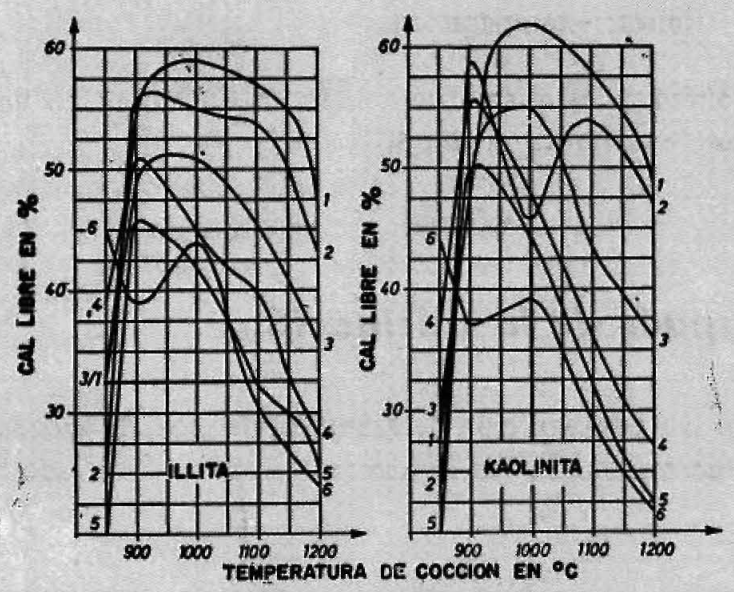

Fig. 1.- CaO libre en función de la temperatura de calcinación y de la finura.

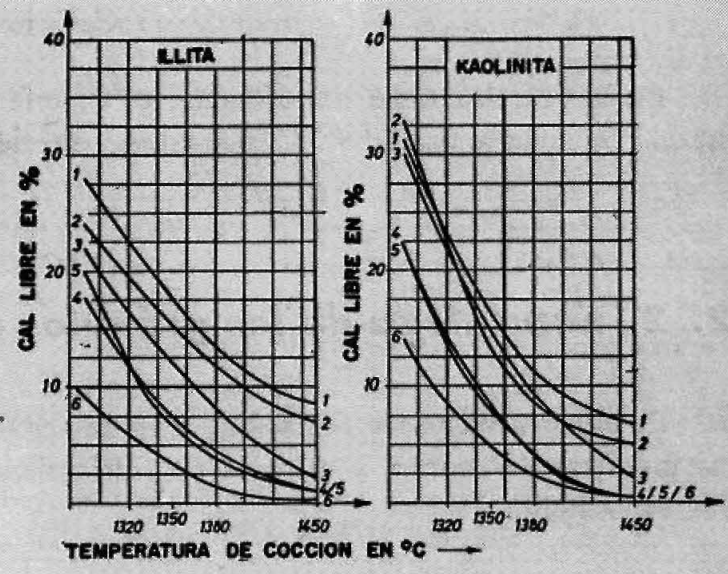

Fig. 2. - CaO libre en función de la temperatura de calclnación $y$ de le finure.

1. El contenido en $\mathrm{CaO}$ libre aumenta fuertemente entre $850^{\circ} \mathrm{C}$ y $900^{\circ} \mathrm{C}$, del $30 \%$ aproximadamente, a más del $50 \%$. Esto corresponde al proceso de descarbonatación, como se ha demostrado también por análisis térmico-diferencial.

2. El final de la descarbonatación ocurre para las mezclas más finas a unos $930^{\circ} \mathrm{C}$, y para las más gruesas a unos $960^{\circ} \mathrm{C}$. Como es sabido, a esta temperatura ha comenzado ya la formación de los minerales del clínker. A pesar de esto, el contenido en cal libre del $40 \%$ hasta el $60 \%$ es muy alto, e indica que entre $900^{\circ} \mathrm{C} \mathrm{y} 1.000^{\circ} \mathrm{C}$ están combinados únicamente de un $8 \%$ aproximadamente a un $28 \%$ de $\mathrm{CaO}$ con $\mathrm{Al}_{2} \mathrm{O}_{3}$ ó $\mathrm{SiO}_{2}$.

3. La curva del contenido de cal libre de la mezcla con la finura 6 demuestra al principio otra tendencia distinta a las demás curvas. El alto contenido en cal libre a $850^{\circ} \mathrm{C}$ indica una descarbonatación muy temprana, sin que esta aportación de $\mathrm{CaO}$ contribuya inmediatamente a la formación de los minerales. En cambio ha tenido lugar, de forma ostensible, a unos $900^{\circ} \mathrm{C}$ y más, una intensa combinación de $\mathrm{CaO}$.

4. La influencia de la finura y la temperatura es aproximadamente la misma. El contenido en $\mathrm{CaO}$ libre disminuye por término medio con la finura, de los más "gruesos" a los más "finos", al $20 \%$, y con temperaturas por encima de $1.300^{\circ} \mathrm{C}$, desde el 15 al $8 \%$.

5. La influencia del tipo de mineral arcilloso se limita a la combinación de la cal, que tiene lugar algo más rápidamente (por encima de unos $1.000^{\circ} \mathrm{C}$ ) para las muestras que contienen caolín, frente a aquellas que contienen illita. 
6. En todos los intervalos de temperatura, el contenido en $\mathrm{CaO}$ libre disminuye con el tamaño de la piedra caliza, de tal forma que las curvas de la mezcla más fina y más gruesa indican el intervalo de la cal libre.

7. El módulo de alúmina (MF) no ejerce ninguna influencia en el contenido en $\mathrm{CaO}$, de forma tal que pudieron deducirse los resultados de los ensayos de las mezclas con distinto MF mediante la obtención de un valor medio de las curvas representadas.

Los resultados de las investigaciones permiten hacer consideraciones en relación con la combina. ción triangular:

$$
\text { contenido en } \mathrm{CaO} \text { libre - finura - temperatura. }
$$

En la práctica tiene importancia, p. e., que se obtenga un mismo contenido en $\mathrm{CaO}$ libre en una mezcla finísima a unos $100^{\circ} \mathrm{C}$ menos que en mezclas de la finura media 3.

\section{2. naturaleza de los gránulos después de la calcinación}

En las pruebas de calcinación, y en relación con la desintegración de gránulos, después de sacadas las muestras del horno, y durante su enfriamiento, fueron hechas las siguientes observaciones, anotadas en la figura 3 :

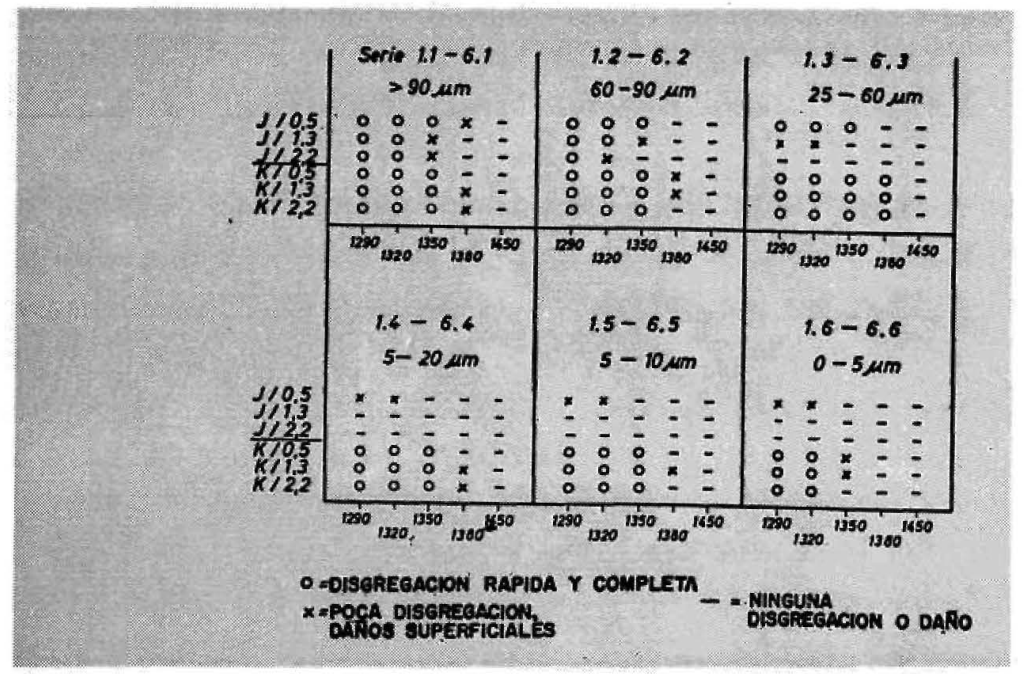

Fig. 3.-Estabilidad de los gránulos.

1. Todos los gránulos del intervalo de calcinación a $1.200^{\circ} \mathrm{C}$ y por debajo, permanecieron intactos (sin daño superficial alguno).

2. Por encima de $1.200^{\circ} \mathrm{C}$ la desintegración de los gránulos se retrasó, tanto al elevar la temperatura como al aumentar la finura.

3. Las muestras con caolín se disgregan mucho más que aquellas preparadas con illita.

4. La dependencia de la disgregación de gránulos con el módulo de alúmina es pequeña. Aumenta algo al crecer dicho módulo. 
La disgregación de los gránulos se relaciona directamente con la transformación $\beta \rightarrow \gamma-\mathrm{C}_{2} \mathrm{~S}$. En las muestras con illita hay que atribuir su menor disgregación a la mayor estabilización del $\beta-\mathrm{C}_{2} \mathrm{~S}$, debido al mayor contenido de álcalis.

La tendencia a la disminución de la desintegración, al aumentar la finura, hay que atribuirla a la mayor formación de alita, y al mismo tiempo, al menor contenido en $\mathrm{C}_{2} \mathrm{~S}$.

La absoluta durabilidad de los gránulos por debajo de $1.200^{\circ} \mathrm{C}$, y a pesar de la formación de $\mathrm{C}_{2} \mathrm{~S}$, se explica, primordialmente, por el gran volumen de poros, que absorbe el aumento de volumen con la transformación del $\mathrm{C}_{2} \mathrm{~S}$.

\section{3. investigaciones microscópicas}

\section{3. 1. determinación de los minerales de clínker}

La determinación cuantitativa de los minerales se realizó sobre secciones pulidas, en los intervalos de temperatura de $1.350^{\circ} \mathrm{C}, 1.380^{\circ} \mathrm{C}$ y $1.450^{\circ} \mathrm{C}$, según el método de recuento planimétrico (5).

Para enjuiciar el progreso de la reacción, se tomó como referencia el contenido de alita, en el intervalo de temperatura entre $1.350^{\circ} \mathrm{C}$ y $1.450^{\circ} \mathrm{C}$.

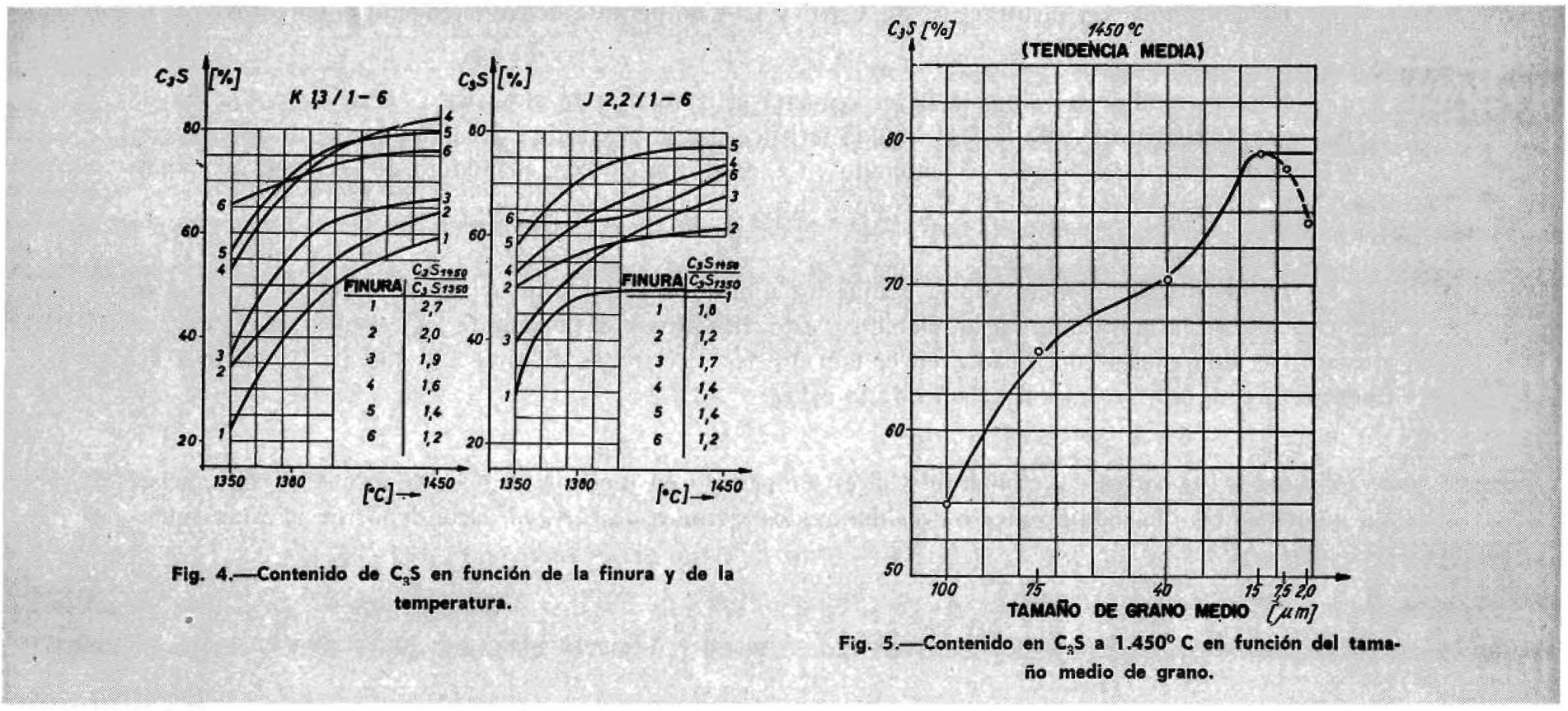

1. En principio, el contenido en alita aumenta sistemáticamente con la temperatura de calcinación, y con la finura, como muestra la figura 4 para las mezc'as $\mathrm{K}$ 1,3/1-6 e I 2,2/1-6.

2. La formación de alita, que comienza a $1.320^{\circ} \mathrm{C}$, depende mucho del tamaño de la caliza, y se eleva, p. e., a $1.350^{\circ} \mathrm{C}$ con la finura, del $20 \%$ al $65 \%$. A $1.450^{\circ} \mathrm{C}$ el aumento es menor, siendo únicamente del $20 \%$ aproximadamente (del $60 \%$ al $80 \%$ ). Según ello, la mayor formación de alita, en mezclas gruesas, ocurre entre $1.350^{\circ} \mathrm{C}$ y $1.380^{\circ} \mathrm{C}$, pero, en mezclas finas, sucede entre $1.320^{\circ} \mathrm{C}$ y $1.350^{\circ} \mathrm{C}$. 
3. El aumento del contenido de alita, en función de la temperatura, para una finura dada, depende de la velocidad de reacción, que está expresada en la figura 4 como relación entre el contenido en alita, a $1.450^{\circ} \mathrm{C}$, y el contenido en alita a $1.350^{\circ} \mathrm{C}$. Con una finura mayor, disminuye la velocidad de formación. Esto significa un desplazamiento de la mayor formación de alita, con la mayor finura, hacia temperaturas más bajas.

Esta tendencia se ha comprobado en todas las mezclas, y coincide con la disminución del contenido en cal libre.

4. Mezclas con la finưra 6 , a $1.450^{\circ} \mathrm{C}$, muestran un menor contenido en alita que las siguientes más gruesas. En la representación del contenido de alita en función del tamaño medio de la caliza, correspondería con un máximo de alita seguido de una inmediata disminución, como demuestra la f:gura 5.

Esta marcha del contenido en alita pareció tener su origen en las pequeñas oscilaciones en la disposición de la cal de las mezclas. Pero las correspondientes investigaciones no pudieron confirmar esta suposición suficientemente, de forma que es preciso hacer más investigaciones.

5. Los contenidos en $\mathrm{C}_{2} \mathrm{~S}$ y en cal libre disminuyen al crecer la finura y la temperatura.

6. La determinación cuantitativa de $\mathrm{C}_{4} \mathrm{AF}$ y $\mathrm{C}_{3} \mathrm{~A}$ no permite deducir tendencias univocas.

7. Con un módulo de alúmina bajo, aumenta el contenido en alita. Esto se atribuye a la mayor y más temprana formación de la fase líquida ferrítica o a la viscosidad del líquido, que disminuye con el módulo de alúmina. Además, el contenido en $\mathrm{C}_{3} \mathrm{~A}$ disminuye con el módulo de alúmina, quedando más $\mathrm{CaO}$ para combinarse con el $\mathrm{C}_{2} \mathrm{~S}$ y formar alita.

8. La investigación microscópica đemuestra únicamente una influencia del módulo de alúmina en la formación de la masa intersticial. Debido a esto, la forma y el tamaño de los cristales de alita no se atteran. Las diferencias confirmadas en el tamaño de los cristales de alita hay que atribuirlas a la influencia directa e indirecta del tamaño de la caliza.

En todos los cortes del clínker, el $\mathrm{C}_{2} \mathrm{~S}$ está repartido en racimos, y muestra formación de maclas. En muestras, con un módulo medio de alúmina, los cristales de $C_{3} A$, en parte dendríticos, están soldados con Cis AF.

\section{3. 2. constitución de poros y tamaño de los cristales}

El volumen de poros, la formación de los mismos, e incluso el tamaño de los cristales de alita, cambian de forma natural, con la temperatura de calcinación. La determinación cuantitativa microscó. pica del volumen de poros demuestra que no sólo es decisiva la temperatura de calcinación, sino que, en mucho mayor medida, lo es el tamaño đe la caliza. Al disminuir éste, merma también el volumen de poros, como se representa en la tabla 3 y figura 6 para la serie de ensayos I 1,3/1-6. 
T A B L A 3

VARIACION DEL VOLUMEN DE POROS CON LA FINURA

$\begin{gathered}\text { Finure } \\ \text { Núm }\end{gathered}$
1
1
2
3
4
5
6

$\begin{gathered}\text { Fraceión } \\ (\mu \mathrm{m})\end{gathered}$
$90-150$
$60-90$
$25-60$
$5-20$
$5-10$
$0-5$

Volumen de poros

(\%)

80

75

60

45

40

30

Las investigaciones en las otras mezclas han demostrado que el módulo de fundentes $\mathrm{y} / \mathrm{o}$ el tipo de mineral arcilloso no influyen de forma clara en el volumen de poros o en su formación.
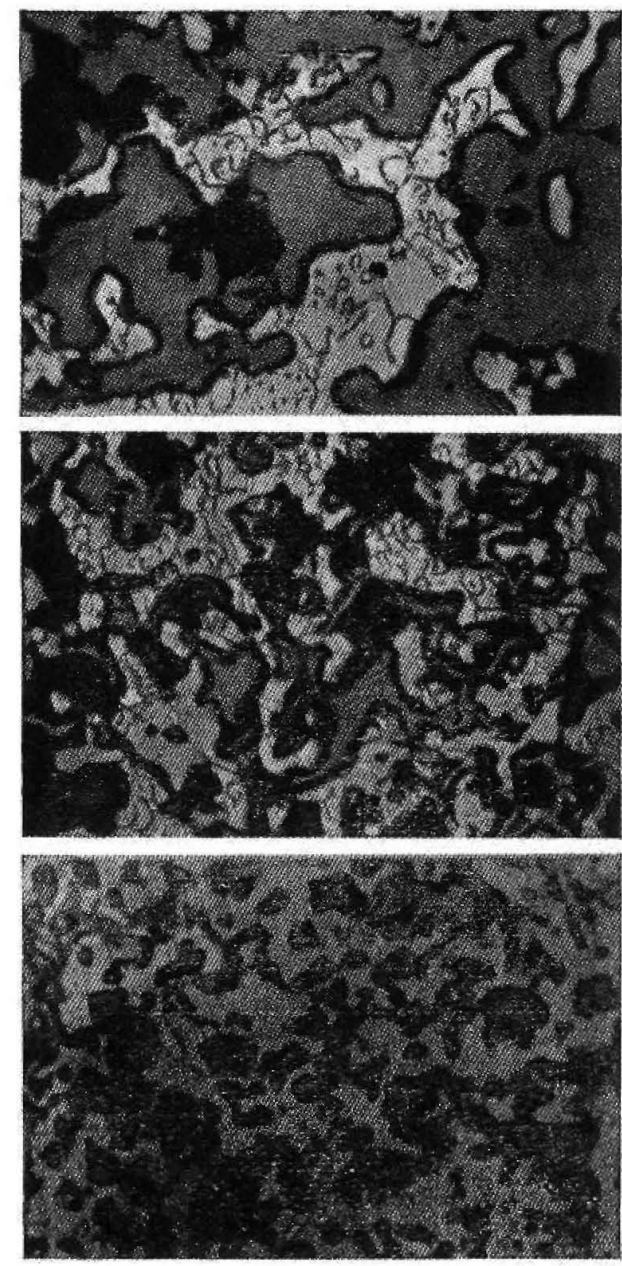

Fig. 6.-Formación de poros a $1.450^{\circ} \mathrm{C}$.
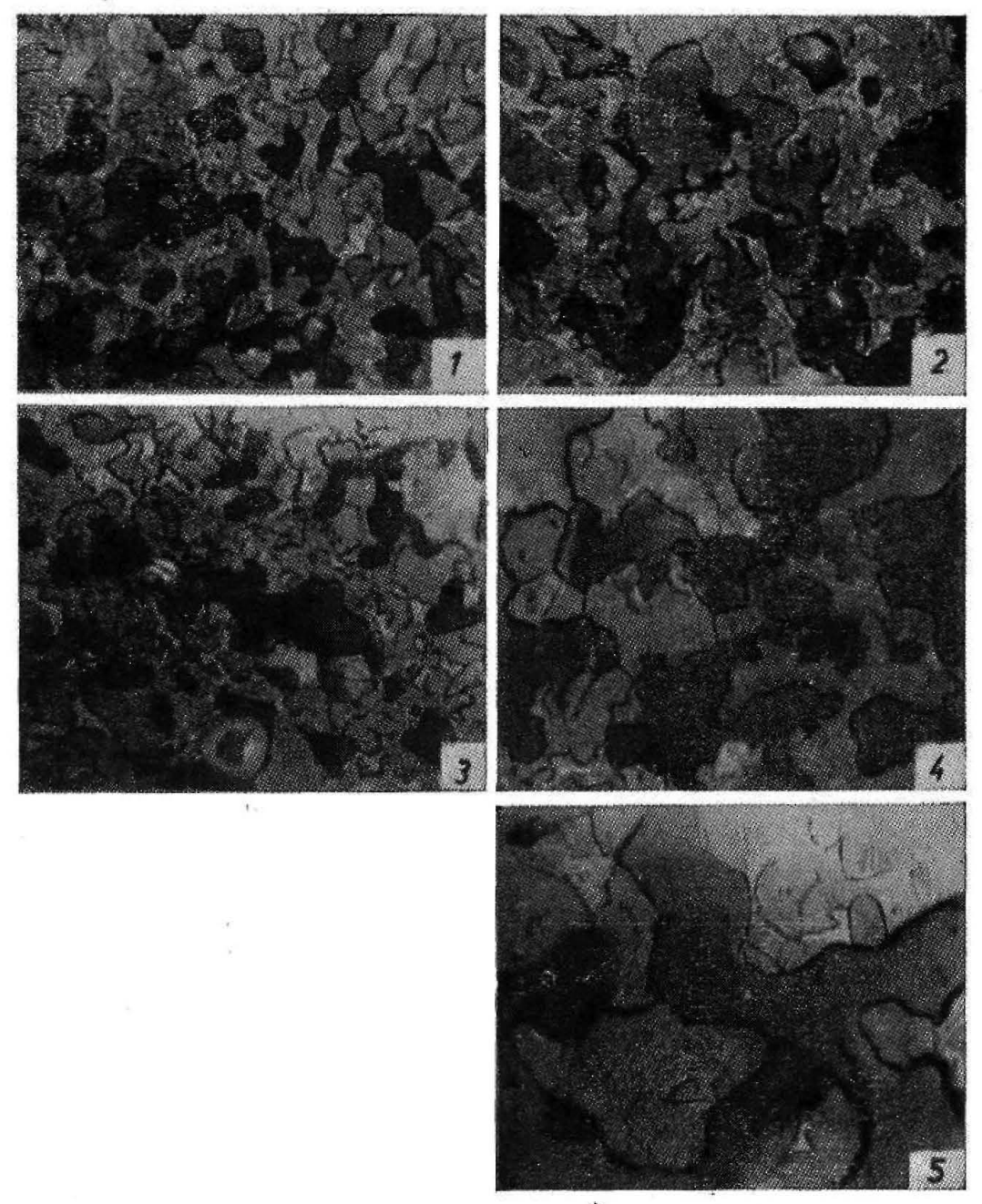

Fig. 7.-Variación del tamaño de los eristales de allta. Númoro de foto/volumen de poros/finura: 1/38/6 ( $1 \%$ de carbón).

$$
\begin{aligned}
& 2 / 66 / 6 \text { (10\% de carbón). } \\
& 2 / 40 / 5 \\
& 4 / 62 / 3 \\
& 5 / 73 / 2
\end{aligned}
$$


Al aumentar el volumen de poros, aumenta también su propio tamaño. Además, los cristales de alita aumentan de tamaño, el contenido en $C_{2} S$ disminuye y los cristales aparecen más frecuentemente aislados y de forma preferentemente idiomorfa.

De esta observación parece resultar, como causa primaria, la variación del tamaño de los cristales de alita. Podría estar influenciada, de forma primaria, por el volumen de poros, mientras que al aumentar la finura, la disminución del volumen de poros llega a ser tan fuerte, que los cristales de alita, que se forman, no tienen ninguna posibilidad de desdoblarse.

Pero como podría suceder también que tuviese influencia directa el tamaño de la caliza, se investigó más detenidamente esta posibilidad. Con este fin se varió, de forma artificiosa, el volumen de poros, añadiendo del 0,4 al $10 \%$ de grafito. Los resultados de los ensayos están resumidos con ejem. plos en la figura 7 .

Con un considerable aumento del volumen de poros—obtenido tan sólo por la adición de carbón-, el tamaño de los cristales de alita permanece constante.

Con ello se ha demostrado que el tamaño de los cristales de alita se influencia directamente con la finura de la caliza.

Al estudiar la influencia de la temperatura de calcinación sobre el volumen de poros, resulta, pues, de interés también la influencia de la finura de la caliza.

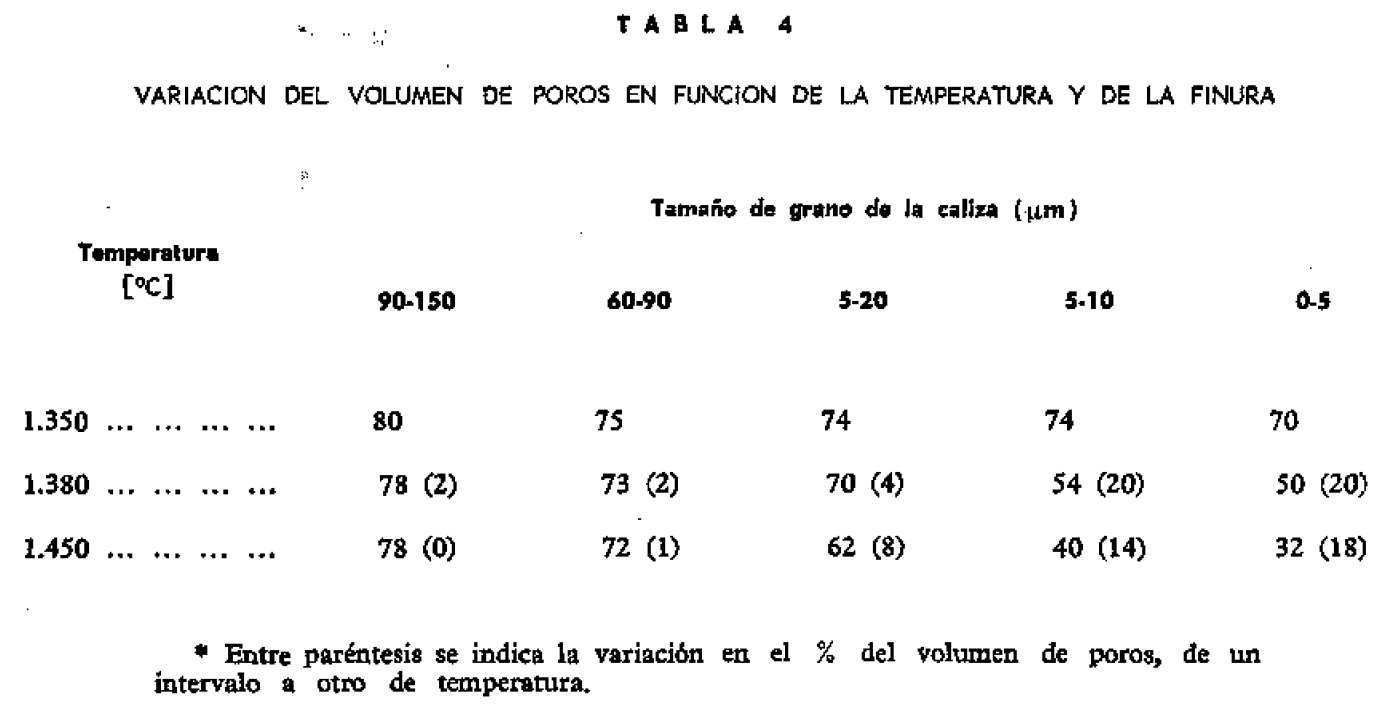

El aumento del volumen de poros en relación con la temperatura y la finura, representado en la tabla 4, muestra la mayor variación del tanto por ciento, entre $1.350^{\circ} \mathrm{C}$ y $1.380^{\circ} \mathrm{C}$, y un considerable aumento, llegando hasta el $20 \%$, con la mayor finura de la caliza. Estas observaciones quizás sirvan también como otra prueba más de la gran influencia de la finura en la marcha de los procesos de reacción. 


\section{4. investigaciones de la estructura fina por rayos $X$}

Las investigaciones cualitativas sobre muestras calcinadas a temperaturas entre $850^{\circ} \mathrm{C} \mathrm{v} 1.450^{\circ} \mathrm{C}$ dieron los siguientes resultados, que se discuten, con respecto a la influencia de la temperatura, del tamaño de grano de la caliza, del módulo de fundentes y del tipo de mineral arcilloso:

1. Al elevar la temperatura aparecen las fases representadas en la figura 8 .

2. El tamaño de grano de la caliza en sus dos finuras extremas se muestra totalmente independiente del módulo de fundentes y del tipo de mineral arcilloso.

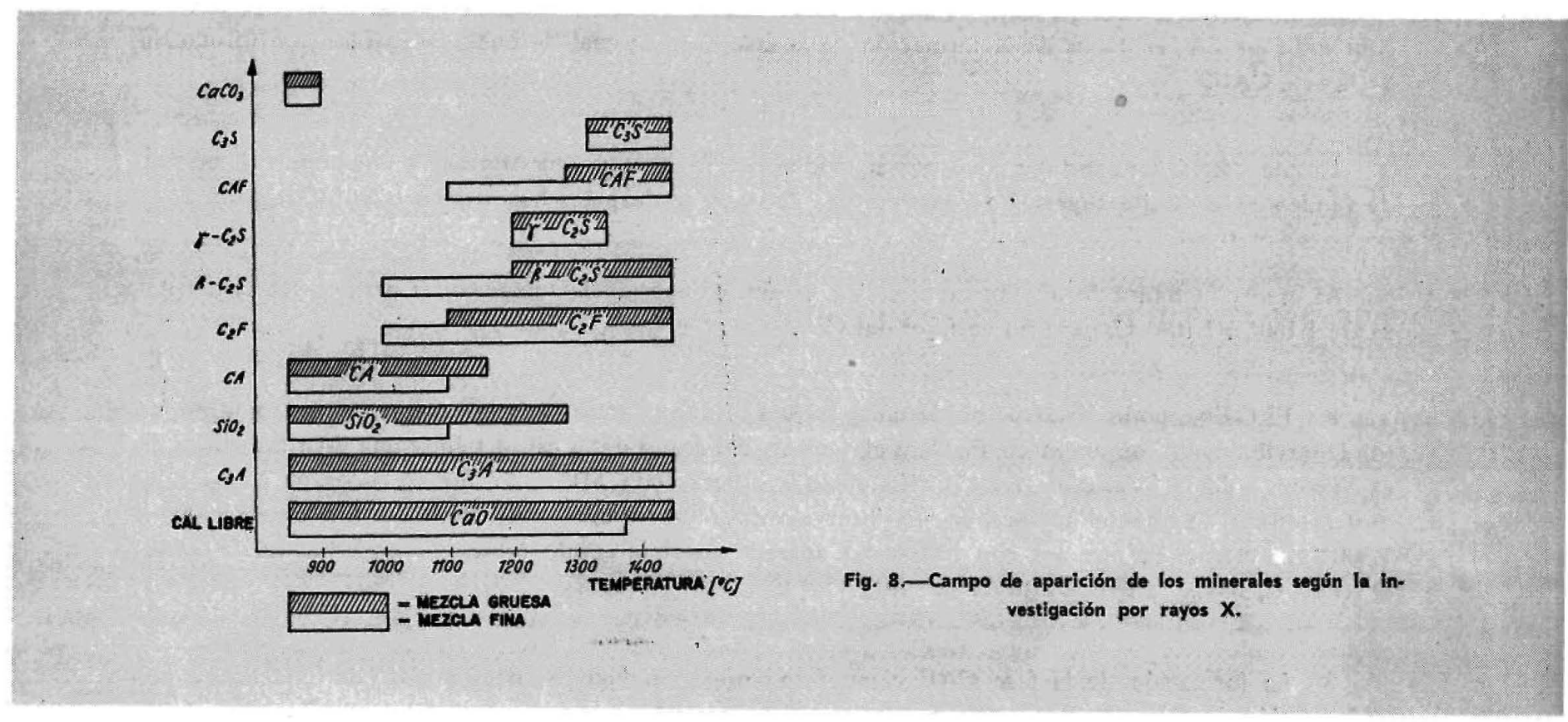

El CaO aparece en todas las mezclas ya a $850^{\circ} \mathrm{C}$, y por rayos $\mathrm{X}$ se observa sólo hasta $1.380^{\circ} \mathrm{C}$, en mezclas finas. El máximo contenido en cal libre se desplaza con el tamaño de grano de la caliza, desde unos $950^{\circ} \mathrm{C}$ hasta $1.100^{\circ} \mathrm{C}$.

Pequeñas desviaciones en los resultados de la determinación de cal libre, según Franke, se deben a las dificultades de la preparación de la muestra. Las diferentes temperaturas de calcinación repercuten fuertemente en el tamaño y dureza de los cristales de $\mathrm{CaO}$, y con ello en la resistencia a la molienda y el peso específico.

3. Independiente de la variación de las magnitudes consideradas, el $\mathrm{CaCO}_{3}$ apareció sólo por debajo de $900^{\circ} \mathrm{C}$.

4. El cuarzo libre no desapareció hasta $\operatorname{los} 1.100^{\circ} \mathrm{C}$, a pesar de la adición de polvo de cuarzo muy fino, o incluso hasta los $1.290^{\circ} \mathrm{C}$ con un tamaño grueso en el grano de caliza.

5. Respecto a la combinación débil del $\mathrm{SiO}_{2}$, a temperaturas inferiores a $1.000^{\circ} \mathrm{C}$, con formación 
del $\beta_{-} \mathrm{C}_{2} \mathrm{~S}$, se puede comprobar por rayos $\mathrm{X}$ que no comienza antes de $1.000^{\circ} \mathrm{C}$; en mezclas gruesas, mucho más tarde, a unos $1.200^{\circ} \mathrm{C}$.

Según varios autores (6), (7), (8) y (9); la formación del $\beta_{-} C_{2} S$ empieza mucho antes, pero entonces exige más tiempo de calentamiento.

6. El $\gamma-\mathrm{C}_{2} \mathrm{~S}$ se observó entre $1.200^{\circ} \mathrm{C}$ y $1.350^{\circ} \mathrm{C}$, y mucho mejor en mezclas que contienen caolín que en mezclas ricas en illita. Esto se atribuye a la mayor estabilización del $\beta$ - $\mathrm{C}_{2} \mathrm{~S}$ por los álcalis de la illita.

7. Independiente de la finura, del módulo de fundentes y del tipo de mineral arcilloso, tanto el CA como el $\mathrm{C} A \mathrm{~A}$, se observaron ya de $850^{\circ}$ a $900^{\circ} \mathrm{C}$. Al elevar la temperatura disminuye primero el contenido en $\mathrm{CA}$, en favor de la formación de la fase $\mathrm{C}_{3} \mathrm{~A}$, la cual después desaparece con formación de la fase CAF.

La zona del CA se influye poco por el módulo đe fundentes; en cambio, al disminuir el módulo de fundentes, el límite superior de temperatura del $C_{1} A$ se desplaza hasta unos $1.200^{\circ} \mathrm{C}-1.290^{\circ} \mathrm{C}$.

Al crecer la finura de molido de la caliza, el límite superior de temperatura para el CA se desplaza de $1.160^{\circ}$ a $1.100^{\circ} \mathrm{C}$, pero no varía el del $\mathrm{C}_{a} \mathrm{~A}$.

8. El $\mathrm{C}_{2} \mathrm{~F}$ se pudo observar desde unos $\mathrm{I} .000^{\circ} \mathrm{C}$, investigando mezclas finas con un módulo de fundentes bajo. El contenido en $\mathrm{Fe}_{2} \mathrm{O}_{3}$ y el tamaño del grano de la caliza tienen una gran influencia en el comienzo de la formación del $\mathrm{C}_{2} \mathrm{~F}$. En mezclas gruesas con $\mathrm{MF}=2,2$ no se encontró el $\mathrm{C}_{2} \mathrm{~F}$; en mezclas finas solamente aparece en un intervalo de temperaturas muy limitado (de unos $1.200^{\circ}$ a $1.300^{\circ} \mathrm{C}$ ), y sólo en mezclas con $\mathrm{MF}=0,5$ aparece en el intervalo total de temperaturas de $1.000^{\circ}$ $61.100^{\circ} \mathrm{C}$, hasta la temperatura final de $1.450^{\circ} \mathrm{C}$.

9. La formación de la fase CAF viene fijada preferentemente por el módulo de fundentes o por la formación de $\mathrm{C}_{2} \mathrm{~F}$.

Al mismo tiempo, con la disminución del contenido en $\mathrm{CA}$ y $\mathrm{C}_{3} \mathrm{~A}$ aparece por primera vez la fase CAF al lado de un máximo de $\mathrm{C}_{2} \mathrm{~F}$, como se representa esquemáticamente en la figura 9, en el ejemplo de la mezcla $\mathrm{I} / \mathrm{O}, 5 / 4$.

Es interesante en este ejemplo el aumento en el contenido de la fase CAF, no comprobándose la existencia de CA ni $\mathrm{C}_{2} \mathrm{~A}$ por encima de $1.300^{\circ} \mathrm{C}$.

Esto conduce a la hipótesis de que por encima de esta temperatura, el aumento en la cantidad de fase $\mathrm{CAF}$ es causada únicamente por la absorción de $\mathrm{C}_{2} \mathrm{~F}$. Esto significaría una variación en la composición de los cristales mixtos, como en efecto se ha podido comprobar. Para una mejor comprobación y poder determinar los ángulos característicos de la fase CAF se extrajeron los silicatos del clínker con ácido salicílico disuelto en metanol, enriqueciéndose el producto en CA y CAF. Para las muestras preparadas con illita, con un bajo módulo de fundentes, la amplitud de los picos disminura continuamente al crecer la temperatura, es decir, se producía un enriquecimiento de la fase CAF en hierro, $\mathrm{y}$ al mismo tiempo una disminución en $\mathrm{CaO}$ y $\mathrm{Al}_{2} \mathrm{O}_{3}$. 
La variación en la composición de las soluciones sólidas se observa en las figuras 9 y 10 . Las investigaciones confirman la formación de la fase $\mathrm{CAF}$ por absorción de $\mathrm{C}_{2} \mathrm{~F}$ en el $\mathrm{C}_{3} \mathrm{~A}$. Forzosamente, el $\mathrm{C}_{2} \mathrm{~F}$ tiene que disminuir entonces con la temperatura.

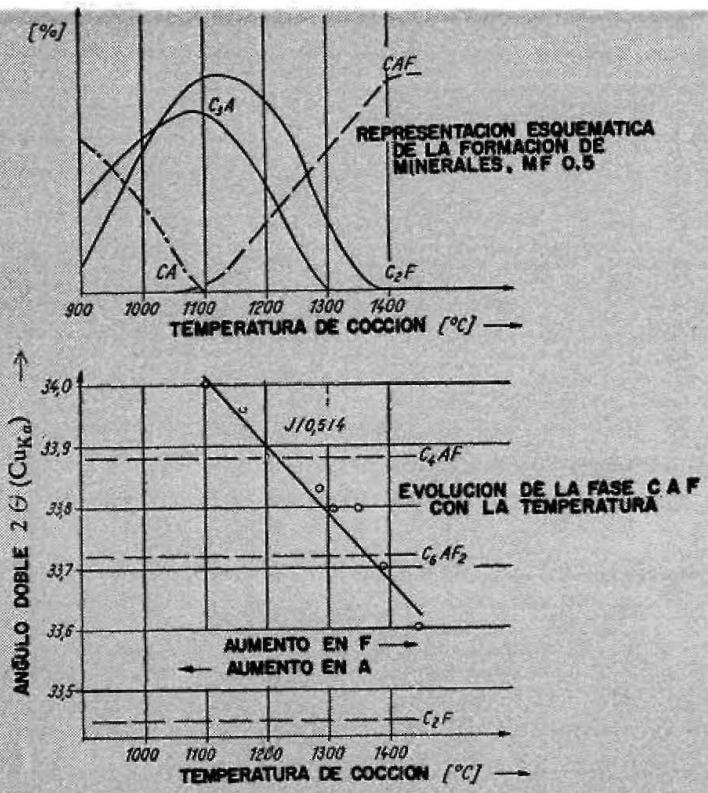

Fig. 9.-Formación de los minerales de elínker.

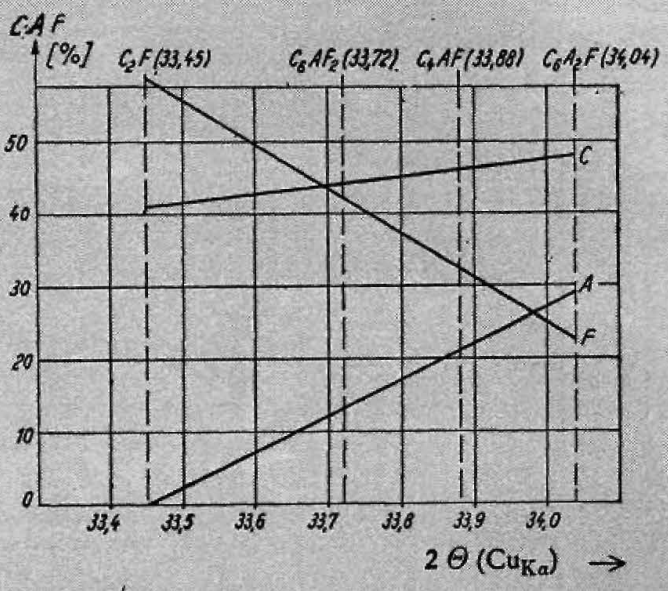

Fig. 10. Composición química de los aluminoferritos éĺcicos.

Además, las investigaciones dan a conocer que la fase CAF en el intervalo de temperaturas de $1.200^{\circ}$ a $1.300^{\circ} \mathrm{C}$ se forma muy intensamente, porque el $\mathrm{C}_{2} \mathrm{~F}$ fundido acelera esencialmente la reacción. Con el mayor tamaño de grano de la caliza se desplazan hacia mayores temperaturas el principio de la formación, el contenido máximo y la disminución de todos los minerales. Esto ocurre también en los contenidos de $\mathrm{C}_{3} \mathrm{~S}$ y $\mathrm{C}_{2} \mathrm{~S}$ determinados microscópicamente, así como en el desarrollo de la combinación de la cal.

\section{5. investigaciones en la resistencia mecánica}

Las probetas calcinadas a $1.380^{\circ} \mathrm{C} \mathrm{y} 1.450^{\circ} \mathrm{C}$ han sido molidas hasta una superficie específica de $3.000 \mathrm{~cm}^{2} \cdot \mathrm{g}^{-1}$, después de la adición de $3 \%$ de anhidrita. En un total de 18 prismas pequeños se determinaron las resistencias a la flexotracción y a la compresión, después de una conservación de 3 , 7 y 28 días. Los valores en las resistencias a flexotracción mostraron la misma tendencia que los de las resistencias a la compresión. Por eso, la representación se limita a los resultados obtenidos en el desarrollo en la resistencia a la compresión, resumidos en las figuras 11 y 12 .

1. En las muestras con caolín, las resistencias en función de la temperatura y del tamaño medio de grano de la caliza corresponden con los contenidos de alita.

Las resistencias dependen tanto del contenido en alita, que ya la variación del módulo de fundentes no tiene un efecto notable. 
2. En las muestras con illita no siempre existe la correspondencia entre los valores de la resistencia y los contenídos en alita. De un modo especial, en las muestras calentadas a $1.450^{\circ} \mathrm{C}$, la influencia del tamaño de grano de la caliza y del contenido de alita sobre el desarrollo de la resistencia no es uniforme.

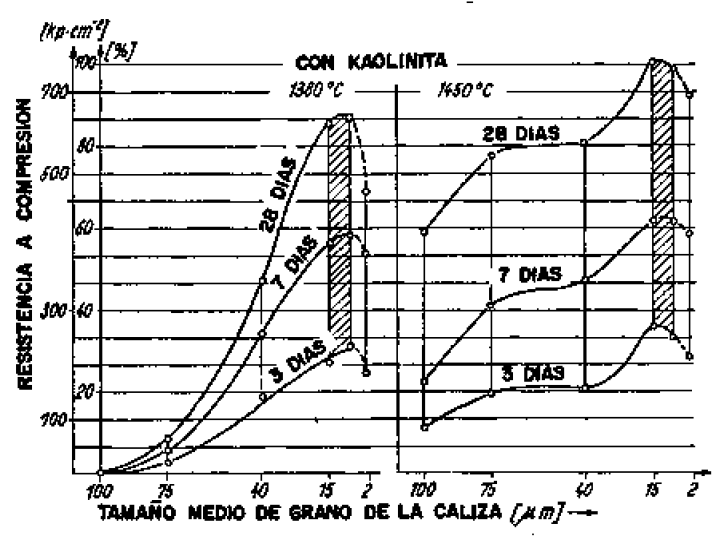

Fit. 11.-Resistencias a la compresión en función del tamaño de grano, de la temperatura $Y$ del período de conservación.

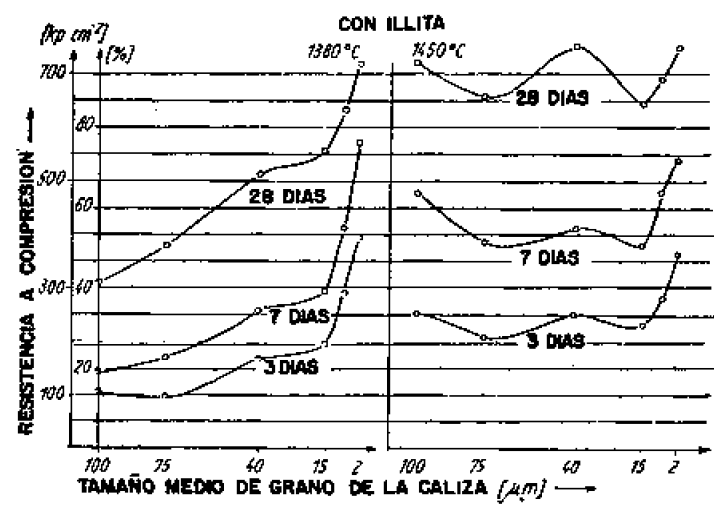

Fig. 12.-Resistencias a la compresión en función del tamafio de grano, de la temperatura $y$ dal período da conservación.

3. En las muestras con illita se ve clara la influencia del módulo de fundentes, pero tampoco en esto se han mostrado tendencias uniformes para justificar unas conclusiones.

Unicamente se puede afirmar con seguridad que las diferencias de resistencia, provocadas por las variaciones en el módulo de fundentes, disminuyen al crecer la finura, la temperatura y el tiempo del ensayo.

Se intentó construir una escala de valores para las distintas magnitudes que influyen en el proceso (finura, temperatura, duración del ensayo, módulo de fundentes y tipo de mineral arcilloso). Es sabido que tales magnitudes se influyen mutuamente, y para poder determinar el efecto de una de ellas, las demás sólo deben variar dentro de unos límites muy estrechos para conocer su influencia.

\section{Análogas consideraciones se pueden hacer en las curvas de la figura 11.}

Por ejemplo, muestra una comparación de las resistencias a $1.380^{\circ} \mathrm{C}$ y $1.450^{\circ} \mathrm{C}$ para finuras comprendidas entre 7,5 y $15 \mu \mathrm{m}$; la elevación de resistencias se expresa en el tanto por ciento del valor máximo.

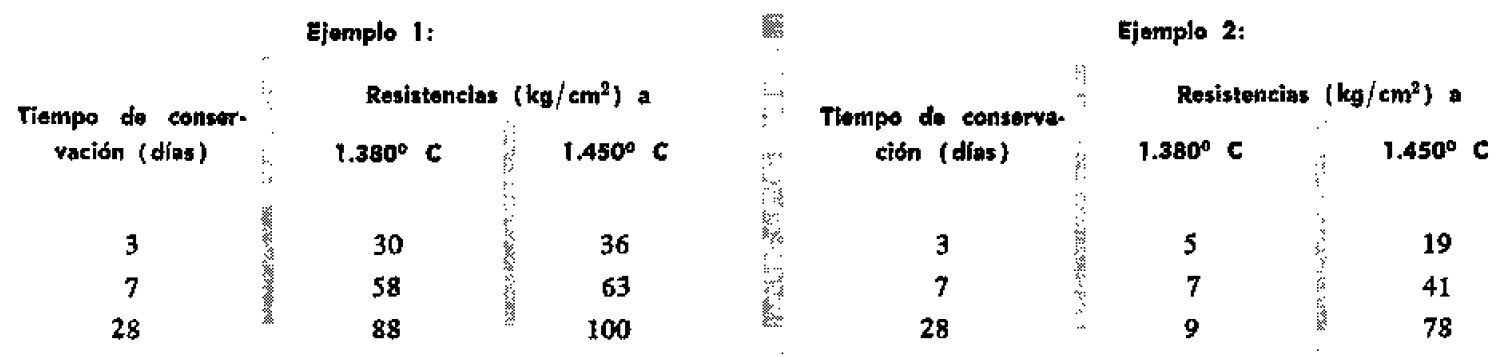


Las resistencias a $1.450^{\circ} \mathrm{C}$ no son esencialmente mucho más altas. Por eso tal vez es suficiente contentarse con temperaturas de calcinación más bajas. Pero esto no es posible con un mayor tamaño de grano de la caliza, porque entonces sería muy grande la disminución de resistencia. Tales condiciones extremadamente desfavorables estarían presentes, p. e., al emplear la finura del ejemplo 2 con un tamaño medio de grano de $75 \mu \mathrm{m}$, obteniendo los siguientes valores.

De estos ejemplos se deduce también la influencia del tiempo de conservación. En el ejemplo 1 es igual para ambas temperaturas, $y$ el aumento de resistencia de una edad a otra es de un tercio, aproximadamente. En cambio, en el ejemplo 2, el aumento de resistencia con el tiempo de conservací́n a $1.380^{\circ} \mathrm{C}$ es muy pequeño, pero a $1.450^{\circ} \mathrm{C}$ es considerable. La formación de los minerales del clínker a $1.380^{\circ} \mathrm{C}$ ha progresado mucho; sólo si las mezclas son finas, de tal forma que el contenido de aiita y las resistencias son altas, y con el tiempo de conservación, las resistencias aumentan considerablemente.

A $1.450^{\circ} \mathrm{C}$ el desarrollo de los minerales del cínker es ya muy fuerte, aun en mezclas gruesas, de tal forma que incluso el aumento de resistencia es aproximadamente igual en todas las finuras.

Del estudio comparativo se deduce que, teniendo en cuenta las circunstancias de fabricación, el orden de influencia de las distintas magnitudes puede ser el siguiente:

$\begin{array}{ll}\text { Influencia } & \text { Finura } \\ & \text { Tiempo de conserpación } \\ & \text { Temperatura de calcinación } \\ & \text { Módulo de fundentes }\end{array}$

Las mejores resistencias se obtienen compaginando los valores de las distintas magnitudes. Inporta conocer tanto la influencia de cada una de las magnitudes como la influencia mutua. Aún no bastan las investigaciones realizadas para poder deducir de ellas conclusiones definitivas en la práctica. Para ello hay que realizar todavía ensayos con mezclas que tengan una curva granulométrica más amplia y que correspondan más a las realidades de la práctica en este aspecto.

Partiendo de las resistencias obtenidas o exigidas, habrá que responder a las cuestiones de la necesidad de una molienda fina de la caliza y de las temperaturas mínimas de calcinación; las consideraciones económicas podrán indicar en qué condiciones habrá que preferir la "molienda más fina" a la mayor temperatura de calcinación.

\section{resumen}

En distintos crudos de cemento se investigó la infuencia del tamaño de grano de la caliza en el desarrollo de la desacidificación a la combinación del $\mathrm{CaO}$ y el contenido en cal libre en el intervalo de temperaturas de $850^{\circ} \mathrm{C}$ a $1.450^{\circ} \mathrm{C}$. La finura de la caliza (se emplearon seis fracciones diferentes, comprendidas entre 0 y $150 \mu \mathrm{m}$ ) tiene una influencia considerable en el desarrollo de la reacción.

La aceleración de los procesos de reacción con la mayor finura se confirmó por la disminución en el contenido de cal libre, por el aumento del contenido de alita observado al microscopio y por medio del análisis por rayos $\mathrm{X}$ en la formación de la fase de aluminatos y de aluminoferritos. El análisis microscópico cuantitativo señaló con el mayor detalle un fuerte aumento del contenido de alita 
en los tres intervalos superiores de temperatura. Al mismo tiempo disminuye el volumen de poros y los propios poros quedan más pequeños.

Además se observó una disminución en el tamaño de los cristales de alita, lo cual no se influencia por el volumen de poros, sino por el tamaño de grano de la caliza. Se atribuye una gran importancia a la influencia de la finura en cuanto al desarrollo de las resistencias. Aunque las investigaciones realizadas tuvieron lugar en condiciones extremas, es apreciable el aumento de resistencia a casi el doble.

Puesto que la magnitud de la influencia de la finura viene también determinada por otras magnitudes y puede ser de importancia la influencia mutua, se realizaron también investigaciones variando el módulo de fundente y empleando caolín o illita como componentes del mineral arcilloso.

Teniendo en cuenta las realidades de la práctica, se puede sacar como conclusión de las investigaciones realizadas, que la influencia de la finura de la caliza es casi tan grande como la de la temperatura.

La influencia del modulo de fundentes en la creación de resistencias es muy pequeña en las muestras con caolín, pero, a veces, muy grande en las muestras con illita.

El orden en la formación de los minerales del clínker se comprobó semi-cuantitativamente por rayos $\mathrm{X}$. Según ello, el $\mathrm{C} A$ aparece ya a partir de $900^{\circ} \mathrm{C}$ al lado del $\mathrm{CA}$, y a mayores temperaturas disminuye en favor de la formación de la fase $\mathrm{CAF}$.

La medición de los picos demostró que se forman cristales mixtos bajo ciertas condiciones, y que a mayor temperatura llegan a ser más ricos en hierro y más pobres en $\mathrm{CaO}$ y $\mathrm{Al}_{2} \mathrm{O}_{3}$.

1. Lehmann, H., Thormann, P.: Die Urtersuchung won Rohstoffen mit Hilfe der kombinierten Dilatometrie und DTA TIZ-Zbl. 86 (1962), S. 606-612.

2. Franke, B.: Freikalkbestimmung. Z, allgem, anorg. Chem. 247 (1941).

3. Keil, F., Gille, F.: Kleinprüfung mit weichem Normenmörtel. Zement 30 (1941), Nr. 40, S, 529-535.

4. Lindner, R.: Silikatbildung durch Reaktion in fesen Zustand. Z. physik. Chem. 6 (1956), S. 129-142.

5. Hennig, A.: Kritische Betrachrunggen zur Volumen- und Oberflächenmessung in der Mikroskopie. Zeiß-Werkzeitschrift (1958), Nr. 30, \$. 78.

6. De Keyser, W. L. Reaktionen in der flüssigen Phase zwischen den Oxiden des Zementsystems. IVA Tidskrift fär Teknisk-Vatenskaplig. Forskung. 26 (1955), S, 292-308.

7. Stegmuller, L, Ney, P.: Reaktionen in festen Zustand beim Erhitzen ron Glimmer-Kalk-Gemischen, Ber. DKG 32 (1955), S. 27-36.

8. Schwiete, H. E: Bindungen des $\mathrm{CaO}$ an die squxe Komponente im natürlichen hochhydraulischen Kalk. Schriftenreihe des Bundesverbandes der deutschehn Kalkindustrie e, V. (1958), H. 3. S. 207-223.

9. Weyer, 1,: Der Verlauf der Resktion und Kaolin und Kalkstein bei statischer Erhitzung. Dissertation Kiel 1930. 